\title{
Cross Species Expression Analysis of Innate Immune Response
}

\author{
Yong Lu ${ }^{1,3}$, Roni Rosenfeld ${ }^{1}$, Gerard J. Nau ${ }^{2}$, and Ziv Bar-Joseph ${ }^{1, *}$ \\ ${ }^{1}$ School of Computer Science, Carnegie Mellon University, Pittsburgh, PA 15213, USA \\ zivbj@Cs. Cmu.edu \\ ${ }^{2}$ Department of Molecular Genetics and Biochemistry, University of Pittsburgh Medical \\ School, Pittsburgh, PA 15213, USA \\ ${ }^{3}$ Present address: Department of Biological Chemistry and Molecular Pharmacology, \\ Harvard Medical School, Boston, MA 02115, USA
}

\begin{abstract}
The innate immune response is the first line of host defense against infections. This system employs a number of different types of cells which in turn activate different sets of genes. Microarray studies of human and mouse cells infected with various pathogens identified hundreds of differentially expressed genes. However, combining these datasets to identify common and unique response patterns remained a challenge. We developed methods based on probabilistic graphical models to combine expression experiments across species, cells and pathogens. Our method analyzes homologous genes in different species concurrently overcoming problems related to noise and orthology assignments. Using our method we identified both core immune response genes and genes that are activated in macrophages in both human and mouse but not in dendritic cells, and vice versa. Our results shed light on immune response mechanisms and on the differences between various types of cells that are used to fight infecting bacteria.
\end{abstract}

Supporting website: http://www.cs.cmu.edu/ lyongu/pub/immune/

\section{Introduction}

Innate immunity is the first line of antimicrobial host defense in most multi-cellular organisms, and is instructive to adaptive immunity in higher organisms [12]. There are multiple types of immune cells, including macrophages, dendritic cells, and others. Depending on their role, each type of cell may respond by activating a different set of genes, even to the same bacteria [5]. In addition to the cell type, innate immune response differs based on the specific pathogen in question [32]. To date, gene expression profiling has been used to investigate transcriptional changes in human and mouse macrophages and dendritic cells during infection with several different pathogens $[5,7,8,13,16,17,22,29,36]$. In each of these studies, a list of genes involved in the response is determined by first ranking the genes based on their expression changes and then selecting the top-ranked genes based on a score or p-value cutoff. While some papers analyze data from multiple cell types or multiple pathogens, a large scale

\footnotetext{
* Corresponding author.
} 
comparison of these datasets across cells, pathogens and different species has not yet been performed.

Microarray expression experiments that study immune response to bacteria infection can be divided along several lines. Here we focus on three such divisions: cell types, bacteria types, and host species.

Innate immunity is the result of the collective responses of different immune cells, which are differentiated from multipotential hematopoietic stem cells [19]. To understand the roles of and possible interplays between different types of immune cells, it is important to identify both the common responses of different immune cells, as well as responses unique to a certain cell type. Identification of genes differentially expressed in macrophages but not in dendritic cells, and vice versa, may highlight their specific functions and help us understand mechanisms leading to their different immune response roles. In addition to the different cells, specific bacteria types are known to trigger very different innate immune responses [32]. Specifically, response to Grampositive and Gram-negative bacteria is activated by different membrane receptors that recognize molecules associated with these bacteria. Finally, many of the key components in the innate immune system are highly conserved [15]. For example, the structure of Toll-like receptors (TLRs), a class of membrane receptors that recognizes molecules associated with bacteria, is highly conserved from Drosophila to mammals. It is less known though to what extent the immune response program is conserved and what other genes play a role in this conserved response.

While each of these subsets of experiments (macrophages vs. dendritic, human vs. mouse etc.) can be analyzed separately using ranking methods and then compared, due to noise in gene expression data methods that rely on a score cutoff become much less reliable for genes closer to the threshold [27]. Thus, analyzing responses to different pathogens and then examining the overlap between the lists derived for each experiment may not identify a comprehensive list of immune response genes. Similarly, while comparing the expression changes triggered by similar bacteria in human and mouse may lead to the identification of conserved immune response patterns, direct comparison of these profiles across experiments is sensitive to noise and orthology assignments, leading to unreliable results and underestimation of conservation [25].

In previous work [26,27] we combined expression datasets from several species to identify conserved cell cycle genes. The underlying idea is that pairs of orthologous genes are more likely than random pairs to be involved in the same cellular system. Thus, if one of the genes in the pair has a high microarray expression score while the other has a medium score, we can use the high scoring gene to elevate our belief in its ortholog, and vice versa. We used discrete Markov random fields (MRFs) to construct a homology graph between genes in different species. We developed a belief propagation algorithm to propagate information across species allowing orthologous genes to be analyzed concurrently.

Here we extend this method in several ways so that it can be applied to analyzing immune response data. Unlike the cell cycle, which we assumed worked in a similar way in all cell types of a specific species, here we are interested in both common responses and distinguishing responses for each dividing factor. This requires a different analysis of the posterior values assigned to nodes in the graph. In addition, for the immune response analysis, genes are represented multiple times in the graph (once for each cell and bacteria type) leading to a new graph topology. We are also 
interested in multiple labels for immune response (up, down, not changing) compared to the binary labels we used for cell cycle analysis. Finally, in this paper we use a Gaussian random field instead of a discrete Markov random field leading to faster updates and improved analysis. Instead of simply connecting genes with high protein sequence similarity, the edges in the graph are determined in a novel way that enables us to utilize the information contained in sequence homology in a global manner, leading to improved prediction performance.

We have used our method to combine data from expression experiments across all three dividing factors. Our method identified a core set of genes containing many of the known immune response genes and a number of new predictions. In addition, our method successfully highlighted differences between conserved responses in macrophages and dendritic cells, shedding new light on the functions of these types of cells.

A number of papers used Markov random field models to integrate biological data sources. These include work on protein function prediction $[6,24]$ and functional orthology prediction [2]. Our method has different goals and uses different data sources. In addition our work differs from these previous papers in several important aspects. Our method propagates information from different cell types and species to improve gene function prediction, while previous work either did not use crossspecies information, or only used it to align networks from different species. We do so by defining our model on a network that explicitly represents genes from different species and cell types. In contrast, previous work either focused on a single species [6,24], or on an aligned network where each node represents an orthologous group [2]. Finally, our model is defined on continuous random variables instead of discrete variables, which enables us to predict three-class labels (up/unchanged/down), while most previous models only handle two-class labels.

\section{Computational Model: Gaussian Random Field}

We formulate the problem of identifying immune response genes using a probabilistic graphical model. In a probabilistic graphical model, random variables are represented by nodes in a graph, and conditional dependency relations are represented by edges. Probabilistic graphical models can be based on directed graphs or on undirected graphs. The model we use here is based on undirected graphs, where special functions (termed "potential functions") are defined on nodes and edges of the graph, and the joint probability distribution is represented by the product of these potential functions. The form of the potential functions encodes our prior knowledge as well as modeling preferences.

We use Gaussian random fields (GRFs) to model the assignment of gene labels. Gaussian random fields are a special type of Markov random fields. In a GRF, every node follows a normal distribution, and all nodes jointly follow a multivariate normal distribution. There are two types of nodes in our graphical model (Fig. 1). The first type is a gene node; it represents the status of a gene in a certain cell type, from a certain host species, in response to a certain type of pathogen. Here we consider two cell types (macrophages and dendritic cells), two host species (humans and mice), and two pathogen types (Gram-negative and Gram-positive bacteria) although the model is general and can accommodate other types as well. The set of possible labels for 


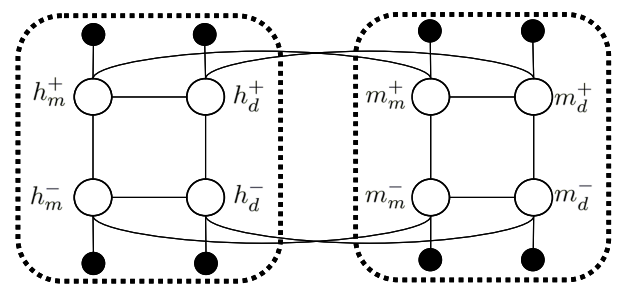

(a)

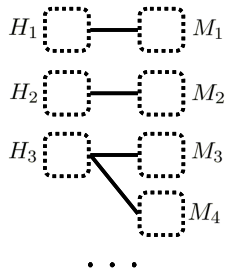

(b)

Fig. 1. Diagram of the Gaussian random field (GRF) model. (a) A subgraph in the GRF containing homologous human and mouse genes. The white node $h_{m}{ }^{+}$represents the (latent) label of the human gene $h$ in macrophages under infection of Gram-positive bacteria. $h_{m}{ }^{-}$represents the gene's label in macrophages under infection of Gram-negative bacteria. $h_{d}{ }^{+}$and $h_{d}^{-}$represent the labels of the same genes in dendritic cells under the infection of Gram-positive or Gram-negative bacteria. $m_{m}^{+}, m_{m}{ }^{-}, m_{d}{ }^{+}$, and $m_{d}^{-}$are similarly defined for the homologous mouse gene $m$. Two white nodes are connected by an edge if they represent the same gene in two experiments, either on the same cell type or under the infection of the same type of bacteria. We also connect two white nodes if they represent homologous genes in the same cell type and under the infection of the same type of bacteria. The black nodes represent the observation from the expression data in a certain cell type and under the infection of the appropriate bacteria. They are connected with the white nodes representing the corresponding genes under the same condition. (b) A high level diagram of the GRF model. Each dotted box represents a subgraph of four nodes related to the same gene as those shown in (a), and each "edge" represents four edges connecting the nodes of homologous genes in the two dotted boxes, in the same way as shown in (a).

each gene can be either two (involved in immune response or not), or three (suppressed, induced, or unchanged during immune response). For simplicity, we will describe our model using binary labels, but will present the results based on both sets of possible labels.

Corresponding to each gene node is a score node, representing the observed expression profile of the corresponding gene. Together, the GRF jointly models the labels of all genes in all cell types, all species, and under both types of infection conditions. The edges in the GRF represent the conditional dependencies between gene labels. We put an edge between two gene nodes when they are a priori more likely to have the same label. Specifically, there are two cases where we add an edge. In the first case, for each gene node in the graph, we connect it with another gene node if the protein sequence similarity between these two genes is high and the experiments related to both nodes are in the same cell and bacteria types. The assumption is that genes with similar sequence are more likely to have similar function in the same type of cell and for the same bacteria. The edge potential function defined on these edges (presented below) introduces a penalty when two genes with high sequence similarity are assigned different labels. In the second case, we connect a gene node with another gene node if the two nodes represent the same gene in the same type of cell or bacteria. Here we assume the genes are likely to function similarly in the same type of cell, or under the same type of infection. Again, the potential function penalizes the case where a gene is assigned different label under different conditions for the same cell. The size of the penalty depends on the strength or weight attached to the edge. Different edges may have different weights (see below). The joint probability is defined as 
the product of the node potential functions and edge potential functions, divided by a normalization function. We can infer the label of individual genes by estimating the joint maximum a posteriori (MAP) assignment of all nodes.

\subsection{Computing the Weight Matrix}

An important issue in random field models is the assignment of edge weights. Employing a similar approach but in a simpler setting, Lu et al. [26] use a Markov random field to jointly model gene statuses in multiple species, where edges in the graph are weighted by BLASTP [1] scores between pairs of genes. Given two genes connected in the graph, the edge weight (BLASTP bit score) represents the sequence similarity between the two genes, which in turn captures the a priori dependency between their labels. While this is a useful strategy, in a Markov random field model edges represent the dependency between the two nodes conditioned on the labels of all other nodes [3]. In contrast, sequence similarity is computed for a pair of genes regardless of other genes. In other words, what a BLASTP score captures is the marginal dependency between the two genes' labels rather than the conditional dependency.

To address this issue we compute new edge weights using the BLASTP score matrix, which captures the marginal covariance of the Gaussian random field. It has been shown that for GRFs the appropriate weight matrix is equal to the inverse of the marginal covariance matrix [39].

Using this observation, we can build a similarity matrix based on BLASTP scores, and use its inverse as the weight matrix for the GRF. Each row (and each column) in the similarity matrix corresponds to a gene. If the BLASTP bit score between two genes is above a cutoff, we set the corresponding elements in the similarity matrix to that score. Otherwise, it's set to zero. We use a stringent cutoff (Results) so that we are fairly confident of the functional conservation when we add a non-zero element. Because the similarity matrix contains scores for all genes in two species, the computational cost to invert it is very high. We thus compute an approximate inverse. We first convert the matrix into a diagonal block matrix by Markov clustering algorithm [9], then compute the approximate inverse by inverting each block independently. The matrix inversion is done using the Sparse Approximate Inverse Preconditioner [14].

Finally, we assign edge weights based on this inverse matrix. Note that each gene is represented by four nodes in the graph, because it is present in different experiments on two cell types and two types of pathogens. For edges connecting gene nodes in different species we set the weight according to the inverse similarity matrix. For edges connecting the same gene in different types of cells and bacteria we use a single hyperparameter as their edge weight for cell and bacteria relationships.

\subsection{Expression Score Distribution}

The gene expression score is a numeric summary computed from the gene's microarray time series, which we will define in Results. We assume that the scores of genes with the same label follow a Gaussian distribution with an experiment specific mean and variance. Due to noise in microarray experiments, these distributions are highly overlapping, making it hard to separate labels by expression score alone. 


\subsection{Node Potential Function}

The node potential functions capture information from gene expression data. For each gene $i$, let $C_{i}$ denote its (hidden) label, $S_{i}$ denote its expression score, $y_{i}$ denote the random variable in the GRF associated with this gene. As mentioned above $C_{i}$ can be a binary variable or a ternary variable if we consider three gene labels. $S_{i}$ and $y_{i}$ are both real variables. Because each $y_{i}$ follows a normal distribution, we need to have a way to link a gene's probability of belonging to each class with the corresponding normal distribution. This is achieved by the probit link function. In the binary labels case let $p_{i}$ be the probability of gene $i$ being an immune response gene conditioned on its expression score $S_{i}$,

$$
p_{i}=\operatorname{Pr}\left(C_{i}=1 \mid S_{i}\right)=\frac{\operatorname{Pr}\left(S_{i} \mid C_{i}=1\right) \operatorname{Pr}\left(C_{i}=1\right)}{\operatorname{Pr}\left(S_{i} \mid C_{i}=1\right) \operatorname{Pr}\left(C_{i}=1\right)+\operatorname{Pr}\left(S_{i} \mid C_{i}=0\right) \operatorname{Pr}\left(C_{i}=0\right)}
$$

For the GRF the node potential function is defined as

$$
\psi_{i}\left(y_{i}\right)=\phi\left(y_{i} \mid \mu=\Phi^{-1}\left(p_{i}\right), \sigma^{2}=1\right)
$$

where $\phi\left(\mathrm{y}_{\mathrm{i}} \mid \mu, \sigma^{2}\right)$ is the probability density function for the normal distribution with mean $\mu$ and variance $\sigma^{2}$, and $\Phi^{-1}(x)$ is the probit function, i.e. the inverse cumulative distribution function for the standard normal distribution. In other words, the information from a gene's expression score is encoded by a normal distribution of $y_{i}$ such that $p_{i}=\operatorname{Pr}\left(y_{i}>0\right)$.

In the case of three labels for genes $\left(C_{i} \in\{-1,0,+1\}\right)$, we can use the following formulas to link the probabilities of $C_{i}$ and $y_{i}$ :

$$
\begin{aligned}
& \operatorname{Pr}\left(C_{i}=1 \mid S_{i}\right)=\operatorname{Pr}\left(y_{i}>1\right), \quad \operatorname{Pr}\left(C_{i}=-1 \mid S_{i}\right)=\operatorname{Pr}\left(y_{i} \leq-1\right) \\
& \operatorname{Pr}\left(C_{i}=0 \mid S_{i}\right)=\operatorname{Pr}\left(-1<y_{i} \leq 1\right)
\end{aligned}
$$

It can be proven (see appendix) that given any (non-zero) probability mass function on $C_{i}$, we can find a normal distribution $N\left(\mu, \sigma^{2}\right)$ such that these formulas are satisfied when $y_{i} \sim N\left(\mu, \sigma^{2}\right)$.

\subsection{Edge Potential Function}

The edge potential functions capture the conditional dependencies between pairs of gene nodes. The assumptions here are that (1) genes with higher sequence similarity are more likely than otherwise to have the same or similar functions; and (2) a given gene is likely to have the same function across cell types and across pathogens.

First we will define the edge potential functions for edges connecting homologous genes in the same cell type and under infection of the same type of bacteria. In this case, the edge potential function depends on the weight matrix we introduced above. Note that although all elements in the BLAST score matrix are non-negative (sequence similarities are non-negative), its inverse matrix may have negative elements. As a consequence, edge weights can be either positive or negative. A positive edge weight indicates that the labels of the two gene are positively correlated, conditioned on the labels of all other gene nodes. A negative edge weight means that they are negatively correlated, conditioned on the other gene nodes. 
The following edge potential function captures this dependency $\left(\lambda_{0}\right.$ is a positive hyperparameter):

$$
\psi_{i j}\left(y_{i}, y_{j}\right)= \begin{cases}\exp \left\{-\lambda_{0}\left|w_{i j}\right|\left(y_{i}-y_{j}\right)^{2}\right\} & \text { if } w_{i j} \geq 0 \\ \exp \left\{-\lambda_{0}\left|w_{i j}\right|\left(y_{i}+y_{j}\right)^{2}\right\} & \text { if } w_{i j}<0\end{cases}
$$

When the edge weight $w_{i j}$ is positive, the edge potential function places a penalty if $y_{i}$ and $y_{j}$ are different. The larger the difference, the higher the penalty. Likewise, when $w_{i j}$ is negative, the edge potential function introduces a penalty based on how close $y_{i}$ and $y_{j}$ are to each other. The penalty becomes higher when we become more confident in $y_{i}$ and $y_{j}$ and the two are close.

For edges connecting the same gene in the same cell type but under infection of different types of bacteria, the edge potential function is defined as

$$
\psi_{1}\left(y_{i}, y_{j}\right)=\exp \left\{-\lambda_{1}\left(y_{i}-y_{j}\right)^{2}\right\}
$$

where $\lambda_{1}$ is a positive hyperparameter. Similarly for edges connecting the same gene under the infection of the same type of bacteria but in different cell types, the edge potential is defined as

$$
\psi_{2}\left(y_{i}, y_{j}\right)=\exp \left\{-\lambda_{2}\left(y_{i}-y_{j}\right)^{2}\right\}
$$

where $\lambda_{2}$ is a positive hyperparameter. Together, the joint likelihood function is defined as

$$
L=\frac{1}{Z} \prod \psi_{i}\left(y_{i}\right) \prod \psi_{i j}\left(y_{i}, y_{j}\right) \prod \psi_{1}\left(y_{i}, y_{j}\right) \prod \psi_{2}\left(y_{i}, y_{j}\right)
$$

\section{Learning the Model Parameters}

In this section we will present our algorithm based on two gene classes. The algorithm can be extended to three gene classes by using different node potential functions (See discussion in Section 2.3). For our model we need to learn the hyperparameters $\lambda$. We also need to learn the parameters of the expression score distributions for each combination of cell types, host species, and pathogen types. In each case, there are four parameters $\left(\mu_{0}, \sigma_{0}{ }^{2}, \mu_{1}, \sigma_{1}^{2}\right)$, i.e. the means and variances of the two different Gaussian distributions, one corresponding to the scores of immune response genes, the other corresponding to the scores of the remaining genes.

We learn these parameters in an iterative manner, by an EM-style algorithm. We start from an initial guess of the parameters. Based on these parameters, we infer "soft" posterior assignments of labels to the genes using a version of the belief propagation algorithm on the GRF. The posterior assignments are in turn used to update the score distribution parameters. We repeat the belief propagation algorithm based on the new parameters to infer updated assignments of labels. This procedure goes on iteratively until the parameters and the assignments do not change anymore. Below we discuss these steps in detail. 
Table 1. Algorithm for combining immune response gene expression data

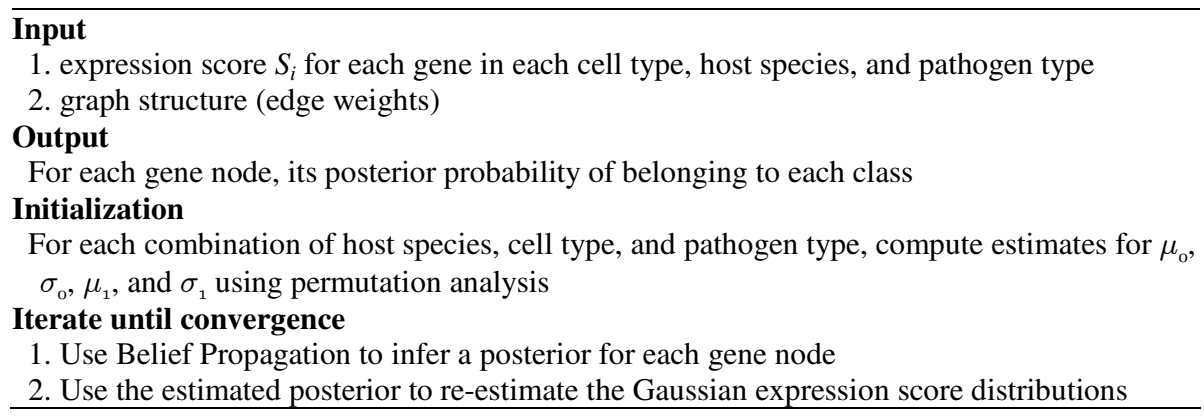

\subsection{Iterative Step 1: Inference by Belief Propagation}

Given the model parameters, we want to compute the posterior marginal distribution for each latent variable $y_{i}$, from which we can derive for each gene node the posterior probability of being involved in immune response. It is hard to compute the posteriors directly because the computational complexity of the normalization function in the joint likelihood function scales exponentially. However, due to the dependency structure in the GRF, we can adapt the standard Belief Propagation algorithm [38] for GRF, and use it to compute all the posteriors efficiently.

Unlike MRFs defined on discrete variables, variables in GRFs are continuous and follow normal distributions. The current estimation of the marginal posterior ("belief") of every latent variable $y_{i}$ in the GRF is a normal distribution. Similarly, the "messages" passed between nodes are also normal distributions.

The Belief Propagation algorithm consists of the following two steps: "message passing", where every node in the GRF passes its current belief to all its neighbors, and "belief update", where every node updates its belief based on all incoming messages. The algorithm starts from a random guess of the beliefs and messages, and then repeats these two steps until the beliefs converge.

(1) Message passing. In this step, every node $y_{i}$ computes a message for each of its neighbors $y_{j}$, sending $y_{i}$ 's belief of $y_{j}$ 's distribution. The message is based on the potential functions, which represent local information (node potential) and pairwise constraints (edge potential), as well as incoming messages from all $y_{i}$ 's neighbors except $y_{j}$.

$$
m_{i j}\left(y_{j}\right) \leftarrow \int \psi_{i j}\left(y_{i}, y_{j}\right) \psi_{i}\left(y_{i}\right) \prod_{k \in N(i) \backslash j} m_{k i}\left(y_{i}\right) \cdot d y_{i}
$$

(2) Belief update. Once node $y_{i}$ has received messages from all its neighbors, it updates the current belief incorporating all these messages and the local information from the node potential. The update rule is as follows

$$
b_{i}\left(y_{i}\right) \leftarrow\left(1 / v_{i}\right) \psi_{i}\left(y_{i}\right) \prod_{k \in N(i)} m_{k i}\left(y_{i}\right)
$$

where $v_{i}$ is a normalization constant to make $b_{i}\left(y_{i}\right)$ a proper distribution. 
Because all the messages and beliefs come from a normal distribution, they can be represented by the corresponding means and variances. Thus, in this case the message update rule and belief update rule above can be formulated into rules updating the means and variances directly, completely avoiding these computationally expensive integration operations. The exact update rules are given in the appendix.

\subsection{Iterative Step 2: Updating the Score Distribution}

The posterior computed in step 1 is based on the current (the g'th iteration) estimation of parameters, collectively denoted by $\Theta^{(\mathrm{g})}$. The goal now is to determine the parameters that maximize the expected log-likelihood of the complete data over the observed expression scores given the parameters $\Theta^{(\mathrm{g})}=\left(\mu_{0}{ }^{(\mathrm{g})}, \sigma_{0}{ }^{(\mathrm{g})}, \mu_{1}{ }^{(\mathrm{g})}, \sigma_{1}{ }^{(\mathrm{g})}\right)$.

To update the parameters of the score distributions, we first compute the posterior probability of a gene being involved in immune response, based on the posterior of $y_{i}$. This is the same as applying the reverse probit function:

$$
\operatorname{Pr}\left(C_{i}=1 \mid \Theta^{(g)}\right)=\int_{0}^{+\infty} b_{i}\left(y_{i}\right) d y_{i}
$$

For simplicity, we use the following notations

$$
p_{i}^{(g)}=\operatorname{Pr}\left(C_{i}=1 \mid \Theta^{(g)}\right) \quad q_{i}^{(g)}=\operatorname{Pr}\left(C_{i}=0 \mid \Theta^{(g)}\right)
$$

The updated distribution parameters for a Gaussian mixture are computed by standard rules

$$
\begin{array}{ll}
\mu_{0}^{(g+1)}=\sum_{i} q_{i}^{(g)} S_{i} / \sum_{i} q_{i}^{(g)} & \mu_{1}^{(g+1)}=\sum_{i} p_{i}^{(g)} S_{i} / \sum_{i} p_{i}^{(g)} \\
\sigma_{0}^{(g+1)}=\sqrt{\frac{\sum_{i} q_{i}^{(g)}\left(S_{i}-\mu_{0}^{(g+1)}\right)^{2}}{\sum_{i} q_{i}^{(g)}}} & \sigma_{1}^{(g+1)}=\sqrt{\frac{\sum_{i} p_{i}^{(g)}\left(S_{i}-\mu_{1}^{(g+1)}\right)^{2}}{\sum_{i} p_{i}^{(g)}}}
\end{array}
$$

Our algorithm is summarized in Table 1.

\subsection{Learning the Hyperparameters}

To learn the hyperparameters $\lambda_{0}, \lambda_{1}$ and $\lambda_{2}$, we use a list of known immune genes. These serve as training data for our algorithm. Following convergence of the belief propagation algorithm we optimize the prediction accuracy using the Nelder-Mead algorithm [33]. Note that this list is not used for the Results below. We divided our list of known immune genes and only used a third to learn the parameters. The other two thirds were used for the comparisons discussed below.

Table 2. Summary of datasets used

\begin{tabular}{|l|c|c|}
\hline \multicolumn{1}{|c|}{ Host/Cell Type } & Gram- Datasets & Gram+ Datasets \\
\hline Human Macrophages & 5 & 2 \\
\hline Human Dendritic Cells & 9 & 2 \\
\hline Mouse Macrophages & 7 & 7 \\
\hline Mouse Dendritic Cells & 7 & 0 \\
\hline
\end{tabular}




\section{Results}

Immune response data. Immune response microarray experiments were retrieved from supporting websites of $[5,7,8,13,16,17,22,29,36]$, totaling 39 data sets. The data sets include experiments on macrophages and dendritic cells in humans and mice. For each cell type we have included experiments using Gram-positive and Gram-negative bacteria, except for mouse dendritic cells, for which we only found Gram-negative bacteria datasets. Human and mouse orthologs were downloaded from Mouse Genome Database [10]. Table 2 summarizes the datasets used in this paper.

Computing expression scores and edge weights. For each gene in each experiment, an expression score is computed from the gene expression time series data. The score is based on the slope of the time series to capture both the change in expression levels and the time between infection and response. Specifically, we first compare the absolute values of the highest and the lowest expression levels. The score is positive if the former is higher, or negative if the latter is higher. Denote the time point that corresponds to the highest absolute value of the expression level as $t_{i}$. The score is computed as follows: $S_{i}=\operatorname{expression}\left(t_{i}\right) / t_{i}$. The score is positively correlated with the height of the peak expression value and increases the earlier this value is reached.

To compute the edge weights we first computed the BLASTP bit score between each pair of protein sequences. We turned the bit scores into a matrix, and set to zero those elements smaller than the 100 (our cutoff). We next computed an approximate sparse inverse of this matrix [14] and used it as the weight matrix for the graph.

Recovering known human immune response genes. To evaluate the performance of our model, we retrieved 642 known human innate immune response genes from [20], and used them as our labeled data. We learned the model parameters by three-fold cross validation using the labeled data. We compared the performance of GRF, MRF, and the baseline model where genes are ranked by their expression score alone. The MRF model is discussed in detail in [26]. We use the fraction of known immune response genes recovered by a model as the performance measure. Because the set of immune response genes we used does not have labels indicating the cell types or infection conditions, we treat a gene as "positive" regardless of the cell type and bacteria type. For GRF and MRF models, the genes were ranked by their highest posterior probability (in any of the cell or bacteria types). For the baseline model, the genes are ranked by their expression scores. As we show in Fig. 2 (a), both GRF and MRF models outperform the baseline model. These models are able to infer a better gene's posterior probability by transferring information between the same gene across cell types or from homologous genes across species. For example, for the top 10\% ranked genes, MRF is able to recover $28 \%$ of known immune response genes, compared with $26 \%$ by the baseline model. Encouragingly, GRF leads to the biggest improvement in performance. Of the top $10 \%$ high scoring genes based on the posterior computed by GRF, 35\% are known immune response genes, a 35\% increase compared to the baseline (score only) model. 


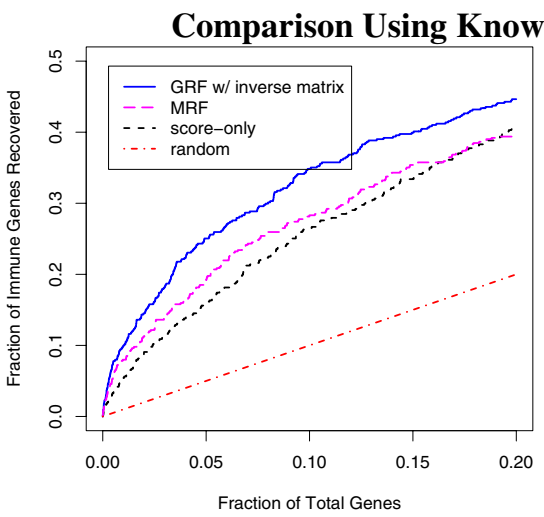

(a)

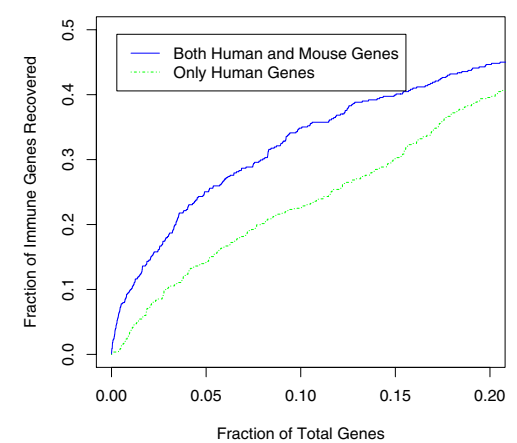

(b)

Fig. 2. (a) Performance comparison of the Gaussian random field (GRF) with improved weights, the Markov random field (MRF), and a baseline model ranking genes by their expression scores. Using MRF we were able to recover $18 \%$ of the known immune genes in the top $5 \%$ of ranked genes. This is a $28 \%$ improvement compared with the baseline model (which recovers $14 \%$ of the immune genes). The GRF model is able to recover $25 \%$ known immune genes at the same threshold, a 79\% improvement over the baseline method and a $38 \%$ improvement over the MRF. (b) Performance comparison of GRF on two different graphs. The first graph contains genes from macrophages and dendritic cells in both human and mouse. The second graph contains genes from human macrophages and dendritic cells, but not from those in mouse. It can be seen that using homology information leads to large improvements.

To study the gain obtained by using cross species analysis we tested the performance of the GRF model when using only the human genes (removing the mouse genes from the graph). As we can be seen in Fig. 2(b), the performance of the GRF when only human genes are included is drastically reduced. The ROC curve when using this data is completely dominated by the curve of the results when using both species, even though the comparison is for recovering known human genes. This indicates that by combining data from both species we can improve the assignment of each species as well.

To determine how sensitive the computed expression cores are to experimental noise, we carried out similar analysis on data that was generated by adding a small Gaussian noise term $\left(\right.$ mean $=0$, variance $=\mathrm{m}^{2}$, where $\mathrm{m}$ is the median expression difference between the first two time points) to each time point of the immune response data. For each of the noise added datasets we determined the score's performance for recovering known human immune response genes as discussed above. We repeated this process 50 times and found that the precision varies by $10 \%$ compared to the real data indicating the robustness of the computed scores (see Supporting website for details).

Identification of common response genes by combined analysis. Based on the learned posterior probabilities, we ranked the genes for each cell type in each species, for both Gram-positive and Gram-negative infections. We identified 57 ortholog pairs for which all nodes for both genes are assigned high posterior (see appendix). These genes are commonly induced by all bacteria in both macrophages and dendritic cells 


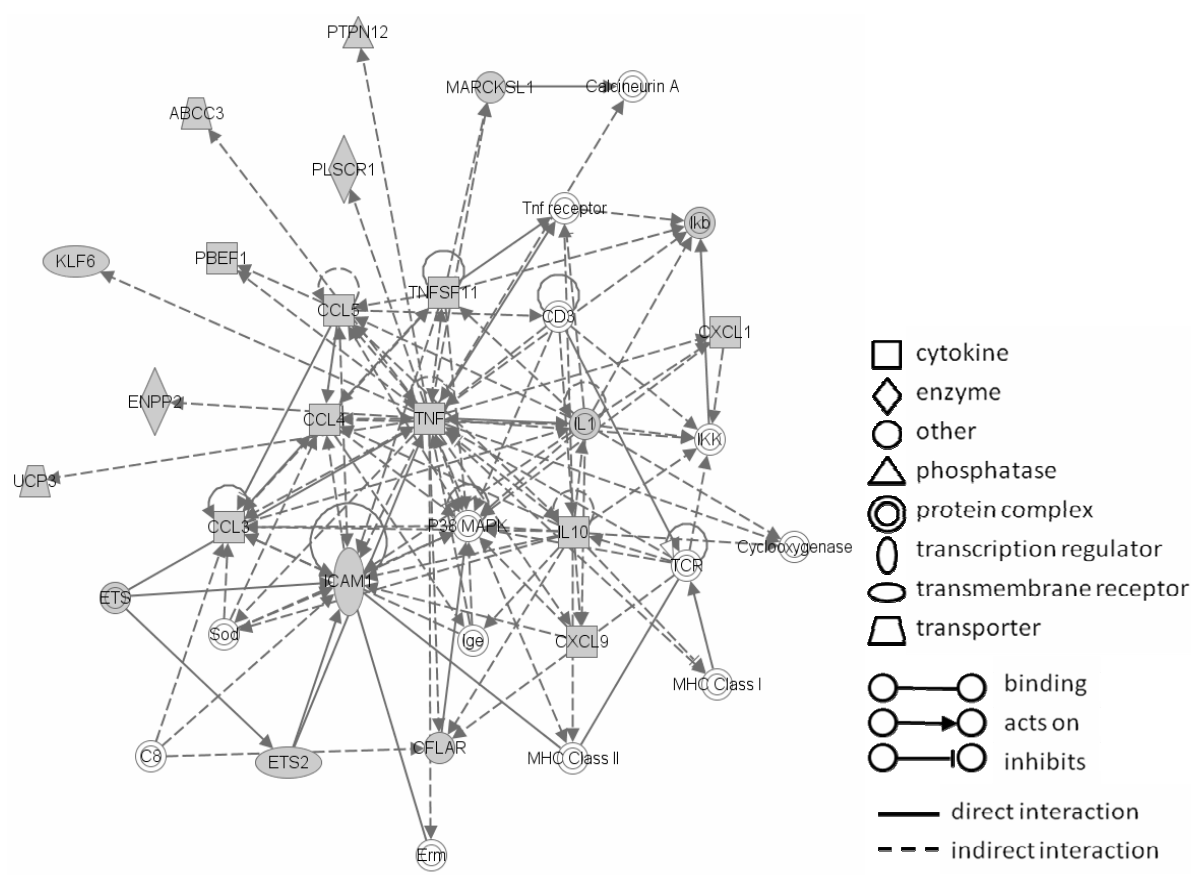

Fig. 3. One of the networks of genes commonly induced in both dendritic cells and macrophages when infected by bacteria, in both human and mouse. The network was constructed using Ingenuity Pathway Analysis (www.ingenuity.com). The gray-colored nodes are genes identified by our method. White-colored nodes are genes interacting with commonly induced genes. Note the large fraction of the pathway recovered by our method. Many known immune response genes are present in this network. IL1 is an important mediator of inflammatory response and involved in cell proliferation, differentiation, and apoptosis (Mizutani et al., 1991; Bratt and Palmblad, 1997). ETS2 is an important transcription factor for inflammation. CCL3, CCL4, and CCL5 are chemokines that recruite and activate leucocytes (Wolpe et al., 1988). The profiles for one of these genes, CCL5, are shown in Fig. 4.

across the two species (Fig. 4). As a sanity check, we first compared our list with a separate list of genes commonly induced in human macrophages by various bacteria. This latter list was derived from expression experiments that were not included in our analysis [32]. The results confirmed the lists we identified. The overlap between the two lists was highly significant with a p-value $=1.70 \times 10^{\wedge}-25$ (p-value computed using hypergeometric distribution).

To reveal the functions of the common response genes we carried out GO enrichment analysis using STEM [11]. The enriched GO categories include many common categories involved in immune responses, including "immune response" (pvalue $=3.9 \times 10^{\wedge}-8$, all $p$-values corrected using Bonferroni), "inflammatory response" (p-value $\left.=2.5 \times 10^{\wedge}-7\right)$, "cell-cell signaling" ( $\mathrm{p}$-value $\left.=1.1 \times 10^{\wedge}-6\right)$, "defense response" $\left(\mathrm{p}\right.$-value $\left.=1.5 \times 10^{\wedge}-6\right)$, and "response to stress" $\left(\mathrm{p}\right.$-value $\left.=2.4 \times 10^{\wedge}-5\right)$. 


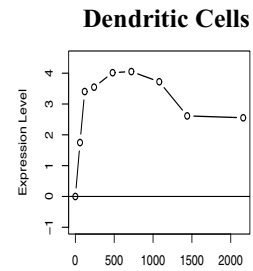

(a) Dendritic Cells

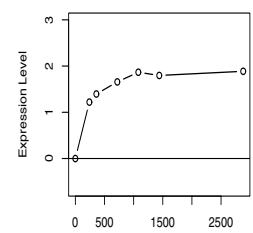

(c)

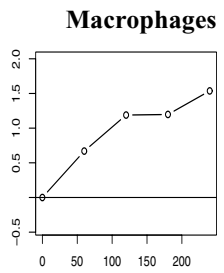

(b) Macrophages

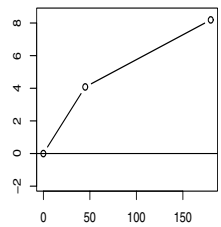

(d)

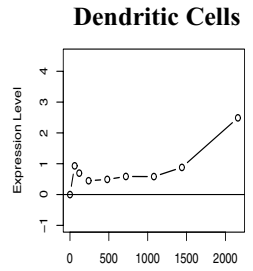

(a)

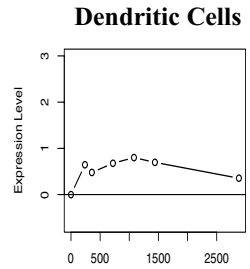

(c)

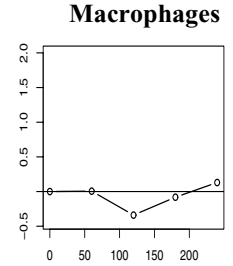

(b)

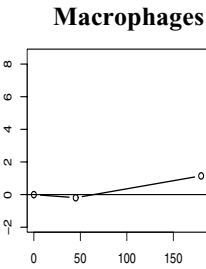

(d)

Fig. 4. Expression profiles of CCL5 identified by our method as a common immune response gene. (a) and (b) expression profiles for human CCL5 in dendritic cells and macrophages. (c), (d) expression profiles for mouse CCL5 in dendritic cells and macrophages. Expression of both genes is strongly induced following infection.

Fig. 5. Expression profiles of CD86 identified to be activated only in dendritic cells. (a) and (b), expression profiles for human CD86 in dendritic cells and macrophages. (c), (d) expression profiles for mouse CD86 in dendritic cells and macrophages. For both species, the expression of CD86 is induced in dendritic cells, but unchanged following infection in macrophages (and only mildly induced at the end of the time course).

Our list recovered many of the classic players of innate immune activation and inflammation. For example, TNF is a pro inflammatory cytokine and stimulates the acute phase reaction [28]. IL1 is an important mediator of inflammatory response and involved in cell proliferation, differentiation, and apoptosis [4,31]. The list also includes chemokines that recruit and activate leucocytes (CCL3, CCL4, CCL5, CXCL1) [37] or attracts T-cells (CXCL9) [35]. Also important to the regulation of inflammation response is IL10, a well-known anti-inflammatory molecule [21]. Additionally, ETS2, $\mathrm{NFkB}$, and JUNB are all very important transcription factors that are activated in inflammation [34]. In addition to recovering genes labeled in the IRIS database [20], which accounts for $21 \%$ of our predictions, we also successfully identified many immune response genes that were not included in the labeled dataset. Six out of the top 10 such genes are known to be commonly induced in host response in macrophages and dendritic cells [18], including PBEF1, an inhibitor of neutrophil apoptosis [23], and MMP14, an endopeptidase that degrades various components of the extracellular matrix [30]. See supporting website for complete list.

To identify the pathways involved in common immune response, we searched for networks enriched by common response genes using Ingenuity Pathway Analysis. One of these networks is shown in Fig. 3.

Immune responses conserved in specific cell types. In addition to genes commonly induced across all dividing factors, we also identified genes that are differentially 
expressed between the two cell types. We identified 127 genes that are highly induced in dendritic cells in both bacteria types across human and mouse, but are not induced in macrophages (Fig. 5). GO enrichment analysis highlights some of the important characteristics of this set of genes, including "cell communication" ( $p$-value $\left.=1.7 \times 10^{\wedge}-10\right)$ and "signal transduction" ( $\mathrm{p}$-value $=1.1 \times 10^{\wedge}-9$ ). (See supporting website for the complete lists.) Many of the genes are known to be associated with functions of dendritic cells, especially antigen processing and presentation. For example, components of the proteosome are prominently represented in the genes determined to be induced in dendritic cells. The proteosome is a necessary first step in MHC class I antigen presentation, a major function of dendritic cells. Peptides generated by the proteosome are then transported from the cytosol to endoplasmic reticulum by TAP, also represented in the gene list, where they are loaded on to MHC I molecules. Antigen presentation by DC is also accomplished through the class II pathway and the DC-specific gene list includes HLA-DRA, a human MHC II (class II) surface molecule. In addition to peptide-MHC complexes, $\mathrm{T}$ cell activation during antigen presentation requires a second signal. CD86, identified as a dendritic cell gene by our algorithm is an essential co-stimulatory molecule that delivers this second signal and is also a marker of dendritic cell maturation. Also in this list are TNFSF9 and TNFSF4, two cytokines that play a role in antigen presentation between dendritic cells and $\mathrm{T}$ lymphocytes.

We have also identified 157 genes that are more likely to be induced in macrophages than in dendritic cells. Among these genes, FNGR1 is important for macrophages to detect interferon-gamma (also known as type II interferon), a key activating cytokine of macrophages. HMGB1, a chromatin structural protein, is believed to be involved in inflammation and sepsis. Another interesting gene is ADAM12, which is from a family of proteinases that are likely involved in tissue remodeling/wound healing by macrophages.

\section{Conclusions and Future Work}

By combining expression experiments across species, cell types and bacteria type we were able to obtain a core set of innate immune response genes. The set we identified contained many of the known key players in this response and also included novel predictions. We have also identified unique signatures for macrophages and dendritic cells leading to insights regarding the set of processes activated in each of these cells types as part of the response.

While our method assumes that homologous genes share similar functions, it is still sensitive to the observed expression profiles. Thus, if two homologs display different expression patterns they would be assigned different labels. Still, homology information is a very useful feature for most genes. Relying on homology information we were able to drastically improve the recovery of the correct set of genes.

While we have focused here on immune response, our method is general and can be applied to other diseases or conditions. We would like to further explore the lists derived by our method to determine the interactions and mechanisms leading to the activation of these genes in the cells they were assigned to. We would also like to expand our method so that it can better utilize the temporal information available in the microarray data. An additional area to explore is to incorporate other sources of 
information in the construction of the weight matrix. For example, it would be interesting to consider protein domains in addition to sequence similarity when creating the weight matrix.

\section{References}

1. Altschul, S.F., Gish, W., Miller, W., Myers, E.W., Lipman, D.J.: Basic local alignment search tool. J. Mol. Biol. 215, 403-410 (1990)

2. Bandyopadhyay, S., Sharan, R., Ideker, T.: Systematic identification of functional orthologs based on protein network comparison. Genome Res. 16, 428-435 (2006)

3. Bishop, C.M.: Pattern Recognition and Machine Learning, pp. 383-392. Springer, Heidelberg (2006)

4. Bratt, J., Palmblad, J.: Cytokine-induced neutrophil-mediated injury of human endothelial cells. J. Immunol. 159, 912-918 (1997)

5. Chaussabel, D., Semnani, R.T., McDowell, M.A., Sacks, D., Sher, A., Nutman, T.B.: Unique gene expression profiles of human macrophages and dendritic cells to phylogenetically distinct parasites. Blood. 202, 672-681 (2003)

6. Deng, M., Chen, T., Sun, F.: Integrated probabilistic model for functional prediction proteins. J. Comput. Biol. 11, 435-465 (2004)

7. Detweiler, C.S., Cunanan, D.B., Falkow, S.: Host microarray analysis reveals a role for the Salmonella response regulator phoP in human macrophage cell death. Proc. Natl. Acad. Sci., USA 98, 5850-5855 (2001)

8. Draper, D.W., Bethea, H.N., He, Y.W.: Toll-like receptor 2-dependent and -independent activation of macrophages by group B streptococci. Immunol. Lett. 102, 202-214 (2006)

9. Enright, A.J., van Dongen, S., Ouzounis, C.A.: An efficient algorithm for large-scale detection of protein families. Nucleic Acids Res. 30, 1575-1584 (2002)

10. Eppig, J.T., Bult, C.J., Kadin, J.A., Richardson, J.E., Blake, J.A., the members of the Mouse Genome Database Group: The Mouse Genome Database (MGD): from genes to mice-a community resource for mouse biology. Nucleic Acids Res. 33, D471-D475 (2005)

11. Ernst, J., Bar-Joseph, Z.: STEM: a tool for the analysis of short time series gene expression data. BMC Bioinformatics 7, 191 (2006)

12. Fearon, D.T., Locksley, R.M.: The instructive role of innate immunity in the acquired immune response. Science 272, 50-54 (1996)

13. Granucci, F., Vizzardelli, C., Pavelka, N., Feau, S., Persico, M., Virzi, E., Rescigno, M., Moro, G., Ricciardi-Castagnoli, P.: Inducible il-2 production by dendritic cells revealed by global gene expression analysis. Nat. Immunol. 2, 882-888 (2001)

14. Grote, M.J., Huckle, T.: Parallel Preconditioning with Sparse Approximate Inverses. SIAM J. Sci. Comput. 18, 838-853 (1997)

15. Hoffmann, J.A., Kafatos, F.C., Janeway Jr., C.A., Ezekowitz, R.A.B.: Phylogenetic perspectives in innate immunity. Science 284, 1313-1318 (1999)

16. Hoffmann, R., van Erp, K., Trulzsch, K., Heesemann, J.: Transcriptional responses of murine macrophages to infection with yersinia enterocolitica. Cell Microbiol. 6, 377-390 (2004)

17. Huang, Q., Liu, D., Majewski, P., Schulte, L.C., Korn, J.M., Young, R.A., Lander, E.S., Hacohen, N.: The Plasticity of Dendritic Cell Responses to Pathogens and Their Components. Science 294, 870-875 (2001) 
18. Jenner, R.G., Young, R.A.: Insights into host responses against pathogens from transcriptional profiling. Nat. Rev. Microbiol. 3, 281-294 (2005)

19. Keller, G., Snodgrass, R.: Life span of multipotential hematopoietic stem cells in vivo. J. Exp. Med. 171, 1407-1418 (1990)

20. Kelley, J., Bono, B.D., Trowsdale, J.: IRIS: a database surveying known human immune system genes. Genomics 85, 503-511 (2005)

21. Lammers, K.M., Brigidi, P., Vitali, B., Gionchetti, P., Rizzello, F., Caramelli, E., Matteuzzi, D., Campieri, M.: Immunomodulatory effects of probiotic bacteria DNA: IL-1 and IL-10 response in human peripheral blood mononuclear cells. FEMS Immunol Med. Microbiol. 22, 165-172 (2003)

22. Lang, R., Patel, D., Morris, J.J., Rutschman, R.L., Murray, P.J.: Shaping Gene Expression in Activated and Resting PrimaryMacrophages by IL-10. J. Immunol. 169, 2253-2263 (2002)

23. Lee, H.C., Goodman, J.L.: Anaplasma phagocytophilum causes global induction of antiapoptosis in human neutrophils. Genomics 88, 496-503 (2006)

24. Letovsky, S., Kasif, S.: Predicting protein function from protein/protein interaction data: a probabilistic approach. Bioinformatics 19(suppl. 1), i197-i204 (2003)

25. Liu, M., Liberzon, A., Kong, S.W., Lai, W.R., Park, P.J., Kohane, I.S., Kasif, S.: NetworkBased Analysis of Affected Biological Processes in Type 2 Diabetes Models. PLoS Genet. 3, e96 (2007)

26. Lu, Y., Rosenfeld, R., Bar-Joseph, Z.: Identifying Cycling Genes by Combining Sequence Homology and Expression Data. Bioinformatics 22, e314-e322 (2006)

27. Lu, Y., Mahony, S., Benos, P.V., Rosenfeld, R., Simon, I., Breeden, L.L., Bar-Joseph, Z.: Combined Analysis Reveals a Core Set of Cycling Genes. Genome Biol. 8, R146 (2007)

28. Lukacs, N.W., Strieter, R.M., Chensue, S.W., Widmer, M., Kunkel, S.L.: TNF-alpha mediates recruitment of neutrophils and eosinophils during airway inflammation. J. Immunol. 154, 5411-5417 (1995)

29. McCaffrey, R.L., Fawcett, P., O’Riordan, M., Lee, K.-D., Havell, E.A., Brown, P.O., Portnoy, D.A.: From the Cover: A specific gene expression program triggered by Grampositive bacteria in the cytosol. Proc. Natl. Acad. Sci. USA 101, 11386-11391 (2004)

30. Mignon, C., Okada, A., Mattei, M.G., Basset, P.: Assignment of the human membranetype matrix metalloproteinase (MMP14) gene to 14q11-q12 by in situ hybridization. Genomics 28, 360-361 (1995)

31. Mizutani, H., Schechter, N., Lazarus, G., Black, R.A., Kupper, T.S.: Rapid and specific conversion of precursor interleukin 1 beta (IL-1 beta) to an active IL-1 species by human mast cell chymase. J. Exp. Med. 174, 821-825 (1991)

32. Nau, G.J., Richmond, J.F.L., Schlesinger, A., Jennings, E.G., Lander, E.S., Young, R.A.: Human macrophage activation programs induced by bacterial pathogens. Proc. Natl. Acad. Sci. USA 99, 1503-1508 (2002)

33. Nelder, J.A., Mead, R.: A Simplex Method for Function Minimization. Comput. J. 7, 308 $313(1965)$

34. Sun, Z., Andersson, R.: NF-kappaB activation and inhibition: a review. Shock 18, 99-106 (2002)

35. Valbuena, G., Bradford, W., Walker, D.H.: Expression analysis of the T-cell-targeting chemokines CXCL9 and CXCL10 in mice and humans with endothelial infections caused by rickettsiae of the spotted fever group. Am. J. Pathol. 163, 1357-1369 (2003)

36. Van Erp, K., Dach, K., Koch, I., Heesemann, J., Hoffmann, R.: Role of strain differences on host resistance and the transcriptional response of macrophages to infection with Yersinia enterocolitica. Physiol Genomics 25, 75-84 (2006) 
37. Wolpe, S.D., Davatelis, G., Sherry, B., Beutler, B., Hesse, D.G., Nguyen, H.T., Moldawer, L.L., Nathan, C.F., Lowry, S.F., Cerami, A.: Macrophages secrete a novel heparin-binding protein with inflammatory and neutrophil chemokinetic properties. J. Exp. Med. 167, 570 $581(1988)$

38. Yedidia, J.S., Freeman, W.T., Weiss, Y.: Understanding belief propagation and its generalizations. In: Exploring Artificial Intelligence in the New Millennium, pp. 236-239. Morgan Kaufmann Publishers Inc., San Francisco (2003)

39. Zhu, X.: Semi-Supervised Learning with Graphs. Ph.D. Thesis, Carnegie Mellon University, CMU-LTI-05-192 (2005)

\section{Appendix}

\section{Proof of Eq (2)}

In this section we prove that for any positive numbers $a, b$, and $c$ satisfying $a+b+$ $c=1$, there exist real numbers $\mu$ and $\sigma$ such that if $y \sim N\left(\mu, \sigma^{2}\right)$, then

$$
\operatorname{Pr}(y \leq-1)=a, \operatorname{Pr}(-1<y \leq 1)=b, \operatorname{Pr}(y>1)=c .
$$

Here $N\left(\mu, \sigma^{2}\right)$ denotes the Gaussian distribution with mean $\mu$ and variance $\sigma^{2}$.

Proof: Let $\Phi$ denote the cumulative density function of the standard Gaussian distribution $N(0,1)$. Let $u=2 /\left(\Phi^{-1}(a+b)-\Phi^{-1}(a)\right), v=1+u \cdot \Phi^{-1}(a)$. Then $N\left(v, u^{2}\right)$ satisfies the conditions in $\mathrm{Eq}(5)$ as required to prove the claim we made for Eq. (2).

\section{Belief propagation for Gaussian Random Fields}

In Section 3.1, we describe the belief propagation algorithm on a Gaussian random field. We give the message passing and belief update rules in Eq (3) and (4). Because each variable in a GRF follows a Gaussian distribution, these equations can be simplified and lead to very efficient update rules.

Note that the operations carried out in Eq (3) and (4) are multiplication of univariate Gaussian distributions and marginalization of bivariate Gaussian distributions. For multiplication of univariate Gaussian distributions with mean $\mu_{i}$ and variance $\sigma_{i}^{2}$, we have

$$
\prod_{i} \exp \left\{-\frac{\left(x-\mu_{i}\right)^{2}}{2 \sigma^{2}}\right\} \propto \exp \left\{-\frac{\left(x-\left(\sum q_{i} \mu_{i}\right) / \sum q_{i}\right)^{2}}{2\left(\sum q_{i}\right)^{-1}}\right\}
$$

where $q_{i}=1 / \sigma_{i}^{2}$. The resulting product is a Gaussian distribution with the following mean and variance

$$
\begin{gathered}
\mu \leftarrow\left(\sum q_{i} \mu_{i}\right) / \sum q_{i} \\
\sigma^{2} \leftarrow\left(\sum q_{i}\right)^{-1}
\end{gathered}
$$

We can get belief update rules for Eq (4) by substituting $\mu_{i}$ and $\sigma_{i}^{2}$ with the mean and variance of $m_{k i}\left(y_{i}\right)$ and $\psi_{i}\left(y_{i}\right)$, where $k$ belongs to the set of the neighbors of $i$ excluding $j$. 
Next we derive the rules for marginalization of bivariate Gaussian distributions in Eq (3). Let

$$
\begin{gathered}
f_{i j}\left(y_{i}\right) \stackrel{\text { def }}{=} \psi_{i}\left(y_{i}\right) \prod_{k \in N(i) \backslash j} m_{k i}\left(y_{i}\right) \sim N\left(v_{i j}, \rho_{i j}^{2}\right) \\
\psi_{i j}\left(y_{i}, y_{j}\right) \cdot f_{i j}\left(y_{i}\right) \sim N\left(\left(\mu_{i}, \mu_{j}\right), \Sigma_{i j}\right)
\end{gathered}
$$

and

$$
\Sigma_{i j}^{-1}=\left(\begin{array}{ll}
p_{i i} & p_{i j} \\
p_{i j} & p_{j j}
\end{array}\right)
$$

We can compute the mean and variance of message $m_{i j}\left(y_{j}\right)$, which is the result of marginalization of the bivariate Gaussian distribution in Eq (3), by matching the lefthand side (LHS) and right-hand side (RHS) of Eq (6). By expanding the exponent of the RHS of Eq (6), we get

$$
\begin{aligned}
& -\frac{1}{2}\left(\begin{array}{ll}
y_{i}-\mu_{i} & y_{j}-\mu_{j}
\end{array}\right)\left(\begin{array}{ll}
p_{i i} & p_{i j} \\
p_{i j} & p_{j j}
\end{array}\right)\left(\begin{array}{c}
y_{i}-\mu_{i} \\
y_{j}-\mu_{j}
\end{array}\right) \\
= & -\frac{1}{2}\left[p_{i i} y_{i}^{2}+p_{j j} y_{j}^{2}+2 p_{i j} y_{i} y_{j}+\cdots\right]
\end{aligned}
$$

Substituting and expanding the exponent of the LHS of Eq (6), we get

$$
-\frac{1}{2}\left[\left(\alpha_{i j}+r_{i j}\right) y_{i}^{2}+\alpha_{i j} y_{j}^{2}+\operatorname{sign}\left(w_{i j}^{*}\right) \cdot 2 \alpha_{i j} y_{i} y_{j}+\cdots\right]
$$

where

$$
\alpha_{i j}=2 \lambda\left|w_{i j}^{*}\right| \text { and } r_{i j}=1 / \rho_{i j}^{2}
$$

Equating (7) and (8), we can get the following update rules for computing the mean and variance of message $m_{i j}\left(y_{j}\right)$

$$
\begin{gathered}
\mu_{j}=\operatorname{sign}\left(w_{i j}^{*}\right) \cdot v_{i j} \\
\sigma_{j}^{2}=\frac{\alpha_{i j}+r_{i j}}{\alpha_{i j} r_{i j}}=\frac{1}{r_{i j}}+\frac{1}{\alpha_{i j}}
\end{gathered}
$$

\section{Identification of Common Response Genes}

Following convergence of our inference algorithm for the GRF model, we obtained the posterior probability of a gene participating in immune response, for each cell type in each species, for both Gram-positive and Gram-negative infections. Using these posteriors we constructed the list of common response genes by selecting ortholog pairs whose posterior probabilities are higher than 0.5 in all cells, bacteria and species. These genes are up-regulated in response to bacterial infections in all types of experiments we looked at. 